\title{
Generalization of conditioned responding: effects of (autobiographical) memory
}

Bert Lenaert $^{a}$, Bram Vervliet ${ }^{a}$, Koen Schruers $^{b}$, \& Dirk Hermans ${ }^{a}$

a Department of Psychology, University of Leuven, Belgium

b School for Mental Health and Neuroscience, Maastricht University, the Netherlands

Centre for the psychology of learning and experimental psychopathology, KU Leuven

\section{Introduction}

$\square$ Generalization occurs when a conditioned response is elicited by a stimulus different from the original conditioned stimulus (CS). Usually, the strength of the conditioned reaction to novel stimuli decreases with increasing dissimilarity to the CS. This can be visualized as a generalization gradient of which the slope indicates the extent of generalization.

$\square$ In these two studies, generalization is approached from a memory perspective. Riccio and colleagues (1984) argued that a memory representation of a stimulus (e.g. CS) is a complex of attributes (e.g., color, shape, texture, etc.). They hypothesized that forgetting of these attributes might provide an explanation for the flattening of generalization gradients over time.

$\square$ If generalization is indeed based on memory for specific 'attributes', one would predict generalization to be a function of individual differences in memory specificity.

DSo, we hypothesize that individuals low in memory specificity will display more extensive generalization of fear responding to stimuli resembling the CS that is followed by an aversive US

\section{Contingency learning study}

Participants: 38 first-year psychology students (25 + ), who scored either high or low on memory specificity, as measured by the Autobiographical Memory Test (AMT; Williams \& Broadbent, 1986).

Design (between subjects):

\begin{tabular}{lll} 
& Acquisition & Generalization Test \\
\hline Low Memory Specificity & $12 \mathrm{CS}+$ & $2 \mathrm{CS}+$ with neutral US \\
Group & $12 \mathrm{CS}-$ & $6 \mathrm{GSs}$ \\
& & $2 \mathrm{CS}-$ \\
High Memory Specificity & $12 \mathrm{CS}+$ & $2 \mathrm{CS}+$ with neutral US \\
Group & $12 \mathrm{CS}-$ & $6 \mathrm{GSs}$ \\
& & $2 \mathrm{CS}-$
\end{tabular}

Dependent measure: US expectancy (scale from 0 to 10)

\section{Stimulus material}
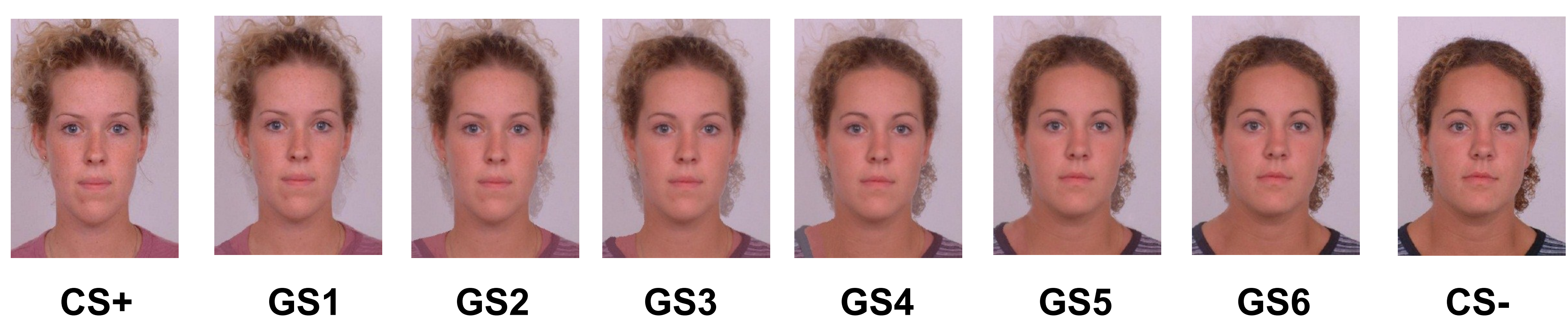

CS+ GS6 GS5

GS4 GS3 GS5
GS2 GS6 CS-
CS+

Results

Conclusions

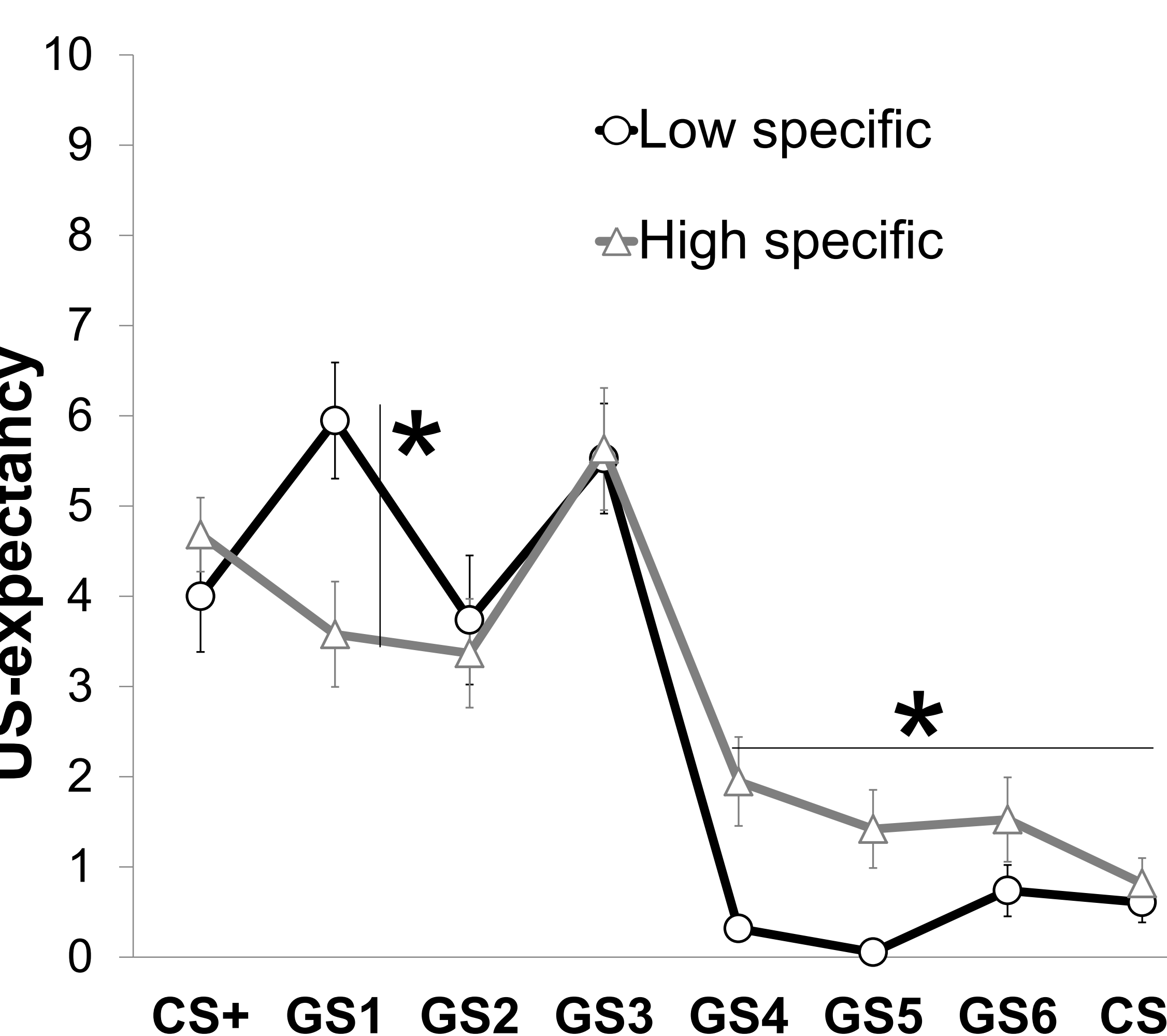

CS+ GS1 GS2 GS3 GS4 GS5 GS6 CS.

$* \mathrm{p}<.05$ Autobiographical memory specificity was significantly associated with the extent of generalization.

- Results are compatible with the theoretical assumption that generalization is a function of memory specificity.

\section{Prospective Fear learning study}

Participants: 378 first year psychology students

Design:

\begin{tabular}{lllll} 
Acquisition & Generalization Test & Re-acquisition & Avoidance test \\
\hline $12 \mathrm{CS}+$ & $2 \mathrm{CS}+$ & $6 \mathrm{CS}+$ & $2 \mathrm{CS}+$ \\
$12 \mathrm{CS}-$ & $2 \mathrm{CS}-$ & $6 \mathrm{CS}-$ & $2 \mathrm{CS}-$ \\
& $8 \mathrm{GSs}$ & & $8 \mathrm{GSs}$
\end{tabular}

Dependent measure:

- Generalization test: US expectancy (scale from 0 to 10)

- Avoidance Test: US could be avoided by hitting the spacebar

- + measure of risk-taking behavior (BART-task)

- + measure of visual memory (RVDLT-task)

Stimulus material

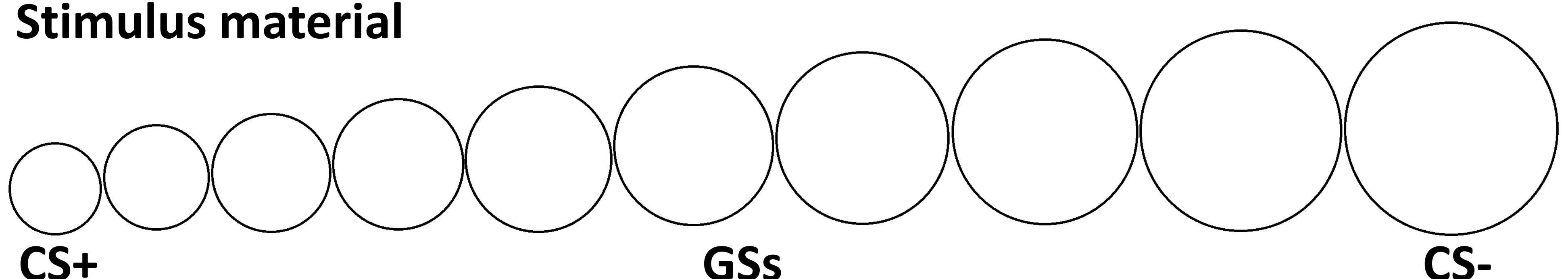
GSs

CS-

US: 9 aversive IAPS pictures selected on arousal ratings (mild, moderate, severe)

Questionnaires

- Depression, Anxiety, Stress Scales (DASS-21)

- Anxiety Sensitivity Index (ASI)

- State and Trait Anxiety Index (STAI)

- White Bear Suppression Inventory (WBSI)

$$
\text { T1 (completed) }
$$

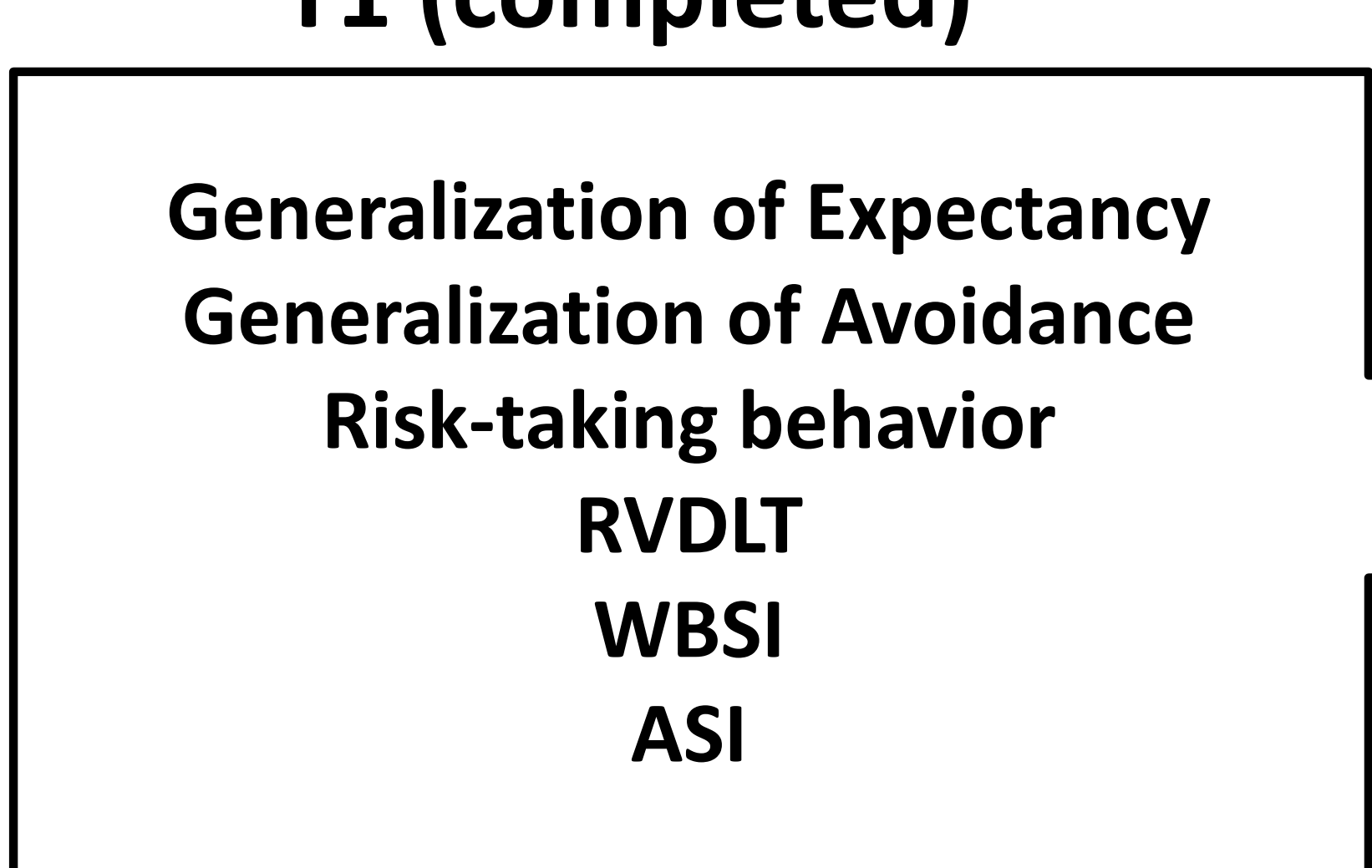

\section{Main hypotheses:}

Generalization of Expectancy/Avoidance predicts the development of anxiety complaints

(Visual) memory is associated with the extent of generalization 